\title{
Non-contrast Cranial Computed Tomography can Change the Emergency Management of Nontraumatic Seizure Patients
}

\author{
Mustafa Sever ${ }^{1, \star}$, Gulsenem Cicek ${ }^{1}$, Necmiye Yalcin Ocak ${ }^{1}$, \\ Yesim Eyler ${ }^{1}$, Rana Toktas ${ }^{1}$, Ibrahim Toker ${ }^{1}$, Murat Yesilaras ${ }^{1}$
}

\author{
${ }^{1}$ Emergency Physician, Department of \\ Emergency Medicine, Tepecik \\ Educational and Research Hospital, \\ Izmir, Turkey \\ *Correspondence \\ adanasever@yahoo.com \\ (Mustafa Sever)
}

\begin{abstract}
Objective: The abnormal cranial computed tomography (CT) findings can change the emergency management in $9-17 \%$ of first seizure patients in the emergency department (ED). The aim is to evaluate the utility of non-contrast cranial CT on emergency management of non-traumatic ED seizure patients. Methods: The patients' charts, who had been coded G-40 according to ICD-10, between 2013 and 2015 were reviewed. All adult patients who applied to the ED with epileptic activity were included. The patients who had trauma, inefficacy in medical record and absence of CT report and detected as not to have seizure were excluded. The socio-demographic and seizure characteristics, CT indications and interpretations and emergency outcomes of patients were investigated. Data were pointed out as numbers, percentages and mean \pm standard deviations. Pearson and Fisher's chi-square tests were used for evaluating of categorical variables. p value $<0.05$ was accepted as significant. Results: 814 patients were included. $55.7 \%$ were male. Mean age was 50 years $(50.50 \pm 20.26$ years, $95 \%$ CI). $56.5 \%$ had chronic and $43.5 \%$ had first seizure activity. New abnormal CT findings were detected in $33.9 \%$ of first and in $11.7 \%$ of chronic seizure patients. Total abnormal CT findings ratio was $52 \% .14 .7 \%$ of first seizure and $7.2 \%$ of chronic seizure patients' emergency managements were changed due to abnormal CT findings. Totally, $10.4 \%$ of all patients' emergency management was detected as changed. Conclusions: In the ED, either in first or in chronic seizure activity, non-contrast cranial CT scan is a practice that may change the patients' emergency managements.
\end{abstract}

\section{Keywords}

Chronic recurrent seizure, Computed tomography, Differential diagnose, Emergency management, First seizure

\section{Introduction}

In the United States (USA), it has been reported that approximately $11 \%$ of the population might have at least one or more seizures during their lifetime, and most of them do not have epilepsy [1]. Intracranial hemorrhages, cerebral lesions, tumors, infections, metabolic abnormalities, or congenital anomalies may be the triggering mechanisms [2]. Mortality has been reported as $1-10 \%$ [3].

Patients with epileptic seizures are recommended to un- dergo neuroimaging to identify structural brain abnormalities that might cause seizures, especially if it is the first seizure, or no clear cause is found [4-8]. Non-contrast Computed Brain Tomography (CT) is the preferred, sufficient, and easily accessible neuroimaging method for the elimination of acute neurological problems requiring immediate intervention $[6,7,9,10]$. It has been reported that the management of $9-17 \%$ of patients presenting to the emergency department (ED) due to first time seizures was changed with non-contrast $\mathrm{CT}$ findings $[7,9,11]$. 
TA B L E 1. Relationship between past medical history and seizure history.

\begin{tabular}{|c|c|c|c|c|}
\hline Past medical history & First seizure n $(\%)$ & Chronic seizure n $(\%)$ & Total n (\%) & p value \\
\hline Cerebrovascular event & $74(20.9)$ & $63(13.7)$ & $137(16.8)$ & 0.006 \\
\hline Malignancy & $49(13.8)$ & $56(12.2)$ & $105(12.9)$ & 0.482 \\
\hline Hypertension & $75(21.2)$ & $13(2.8)$ & $88(10.8)$ & $\mathbf{0}$ \\
\hline Diabetes mellitus & $52(14.7)$ & $13(2.8)$ & $65(8)$ & $\mathbf{0}$ \\
\hline Cranial operation & $16(4.5)$ & $46(10)$ & $62(7.6)$ & 0.003 \\
\hline Chronic Renal Failure & $19(5.4)$ & $11(2.4)$ & $30(3.7)$ & 0.025 \\
\hline Dementia & $20(5.6)$ & $8(1.7)$ & $28(3.4)$ & 0.002 \\
\hline Drug/alcohol abuse & $19(5.4)$ & $8(1.7)$ & $27(3.3)$ & 0.004 \\
\hline Coronary artery disease & $20(5.6)$ & $4(0.9)$ & $24(2.9)$ & $\mathbf{0}$ \\
\hline Mental motor retardation & $5(1.4)$ & $21(4.5)$ & $26(2.9)$ & 0.011 \\
\hline Subarachnoid hemorrhage & $9(2.5)$ & $14(3)$ & $23(2.8)$ & 0.669 \\
\hline Asthma & $12(3.4)$ & $7(1.5)$ & $19(2.3)$ & 0.08 \\
\hline Cerebral palsy & $3(0.8)$ & $10(2.2)$ & $13(1.6)$ & 0.135 \\
\hline Congestive heart failure & $7(2)$ & $2(0.4)$ & $9(1.1)$ & $0.046 *$ \\
\hline Hydrocephalus & $4(1.1)$ & $5(1.1)$ & $9(1.1)$ & 1 \\
\hline Parkinson & $5(1.4)$ & $3(0.6)$ & $8(0.9)$ & 0.305 \\
\hline Psychotic disorder & $5(1.4)$ & $3(0.6)$ & $8(0.9)$ & 0.305 \\
\hline Arrhythmia & $4(1.1)$ & $2(0.4)$ & $6(0.7)$ & 0.412 \\
\hline CNS infection & $0(0)$ & $4(0.9)$ & $4(0.5)$ & 0.137 \\
\hline Liver disease & $2(0.6)$ & $0(0)$ & $2(0.2)$ & 0.189 \\
\hline Trauma & $0(0)$ & $2(0.4)$ & $2(0.2)$ & 0.508 \\
\hline AV malformation & $0(0)$ & $2(0.4)$ & $2(0.2)$ & 0.508 \\
\hline Valve Disease & $1(0.3)$ & $1(0.2)$ & $2(0.2)$ & 1 \\
\hline HIV & $1(0.3)$ & $0(0)$ & $1(0.1)$ & 0.435 \\
\hline Total (n) & 354 & 460 & 814 & \\
\hline
\end{tabular}

AV: Arterio-venous; CNS: Central Nervous System.

Percentage values are the ratio of the row variable to the total number of patients in the column variable.

$P$ values are given as Pearson Chi-Square test values.

The value marked '*' is Fisher's Exact Test data.

Neuroimaging was suggested for the first time in 2011 for the change in seizure character, severe head trauma, prolonged postictal period, newly developed focal neurological deficit, anticoagulant use, ongoing fever, AIDS (Adult Immun Deficiency Syndrome) or other suspected infective conditions in patients presenting to ED with chronic seizure activity $[12,13]$.

This study aimed to evaluate the effect of non-contrast CT imaging used for differential diagnosis on the management of patients in ED with non-traumatic seizure activity (first or chronic seizure activity).

\section{Methods}

\subsection{Study design and setting}

The study was conducted retrospectively in a tertiary academic emergency medicine clinic, which serves approx- imately 180.000 adult patients annually. The study was approved by Izmir Tepecik Educational and Research Hospital Local Ethical Committee (Ethical committee approval date and no; 30.06.2015/28).

\subsection{Selection of participants}

All patients above 18 years of age who were admitted to ED for epileptic seizure activity between January 1, 2013, and May 31, 2015, were enrolled in the study. Patients who had a history of trauma, lacking information on file, who did not have CT imaging, and who were found not to have a seizure were excluded from the study.

\subsection{Methods and measurements}

The information of patients diagnosed with epilepsy (G-40) on the relevant dates was evaluated by using the International Classification of Diseases-10 diagnostic code in the 
TA B L E 2. Distribution of abnormal computed tomography findings according to seizure history.

\begin{tabular}{lcccc} 
CT findings & First seizure n (\%) & Chronic seizure n (\%) & Total n (\%) & p value \\
\hline Chronic infarct sequelae & $84(23.7)$ & $95(20.6)$ & $179(22)$ & 0.293 \\
Diffuse cortical atrophy & $84(23.7)$ & $76(16.5)$ & $160(19.7)$ & $\mathbf{0 . 0 1 3}$ \\
\hline Mass & $40(11.3)$ & $31(6.7)$ & $71(8.7)$ & $\mathbf{0 . 0 2 4}$ \\
\hline Operation sequelae & $14(3.9)$ & $39(8.5)$ & $53(6.5)$ & $\mathbf{0 . 0 1}$ \\
\hline Focal edema & $27(7.6)$ & $23(5)$ & $50(6.1)$ & 0.122 \\
Acute ischemic lesion & $11(3.1)$ & $16(3.5)$ & $27(3.3)$ & 0.77 \\
\hline Shift & $4(1.1)$ & $9(1.9)$ & $13(1.6)$ & 0.351 \\
Hemorrhage & $5(1.4)$ & $5(1.1)$ & $10(1.2)$ & 0.754 \\
\hline Hydrocephalus & $5(1.4)$ & $4(0.9)$ & $9(1.1)$ & 0.513 \\
Diffuse edema & $2(0.6)$ & $0(0)$ & $2(0.2)$ & 0.189 \\
Arachnoid cyst & $0(0)$ & $2(0.43)$ & $2(0.2)$ & 0.508 \\
Toplam (n) & $\mathbf{3 5 4}$ & $\mathbf{4 6 0}$ & $\mathbf{8 1 4}$ & \\
\hline
\end{tabular}

Percentage values are the ratio of the row variable to the total number of patients in the column variable.

$P$ values are Fisher's Exact Test data.

CT: Computed Tomography.

hospital medical registration system. Sociodemographic characteristics (age and sex), seizure characteristics (type, characteristics, duration, history), past medical and antiepileptic medication histories, CT indications and interpretations, and outcomes were recorded in a commercial statistical program.

\subsection{Choosing the reference standards}

CT interpretations were evaluated with the initial version. The definitions of chronic ischemic and chronic hemorrhagic lesions mentioned in the comments were collected under the title of "chronic infarct sequelae." Diffuse edema, hydrocephalus, diffuse cortical atrophy findings were defined as diffuse lesions. Operation sequelae, chronic infarct sequelae, arachnoid cyst, shift, mass, acute ischemic lesion, acute hemorrhage, focal edema were defined as focal lesions. Combined focal and diffuse lesions were defined as mixed lesions.

Indications for CT imaging were accepted as the change in seizure character, prolonged seizure, increase in the frequency of seizures, change in seizure type, and prolonged postictal period. These indications were determined based on contemporary literature [13].

Status epilepticus was accepted as having a seizure activity of more than 30 minutes or continuous seizure or lack of a complete improvement in consciousness between two or more seizure activities as defined by the American Epilepsy Society (AES) in 2016 [14].

Refractory seizures (refractory status epilepticus) were defined as the inability to cease both convulsive and nonconvulsive seizures clinically and electroencephalographically despite benzodiazepine and second antiepileptic drug [12].

In laboratory tests, serum $\mathrm{Na}<135 \mathrm{mmol} / \mathrm{L}$ was defined as hyponatremia, serum $\mathrm{Na}>145 \mathrm{mmol} / \mathrm{L}$ as hyperna- tremia, serum glucose $<70 \mathrm{mg} / \mathrm{dL}$ as hypoglycemia and serum glucose $>110 \mathrm{mg} / \mathrm{dL}$ as hyperglycemia.

The criteria for change in the management of patients in ED was defined as the presence of new abnormal findings requiring hospitalization in non-contrast brain $\mathrm{CT}$.

\subsection{Outcomes}

Sociodemographic and medical characteristics, seizure characteristics, and CT findings were determined as primary outcome while the effects of CT findings on patient outcomes were determined as the secondary outcome.

\subsection{Statistical Analysis}

All statistical analyses were performed using the Statistical Package for Social Sciences ${ }^{\circledR}$ (SSPS) software (SPSS for Windows, Version 22, IBM Inc. ®-USA). The distribution of sociodemographic data was analyzed using Histogram. Pearson and Fisher's chi-square tests and Mann-Whitney-U test were used for comparison of non-parametric variables. Quantitative variables were presented as means \pm Standard deviation, and qualitative variables were expressed as numbers and percentages. All analyses were performed within a $95 \%$ confidence interval, and a $p$-value $<0.05$ was considered statistically significant.

\section{Results}

1129 patients meeting the inclusion criteria were evaluated. A total of 315 patients were excluded from the study, 136 due to the absence of actual epileptic activity, 111 due to trauma, 63 due to lack of information on files, 4 due to the absence of CT imaging, and 1 being under 18 years of age. 814 patients were enrolled in the study. 
TA B L E 3. Distribution of abnormal CT findings according to imaging indications.

\begin{tabular}{|lccccccc|}
\hline CT Findings & CSC n (\%) & FS n (\%) & HBT n (\%) & ND n (\%) & SE n (\%) & Total (n) & p value \\
\hline CIS & $81(20.5)$ & $64(21.8)$ & $7(26.9)$ & $20(36.4)$ & $7(16.3)$ & $\mathbf{1 7 9}$ & 0.079 \\
\hline DCA & $61(15.4)$ & $68(23.1)$ & $10(38.5)$ & $10(18.2)$ & $11(25.6)$ & $\mathbf{1 6 0}$ & $\mathbf{0 . 0 0 9 *}$ \\
\hline Mass & $25(6.3)$ & $30(10.2)$ & $1(3.9)$ & $9(16.4)$ & $6(14)$ & $\mathbf{7 1}$ & $\mathbf{0 . 0 4 1 *}$ \\
\hline Operation sequelae & $34(8.6)$ & $11(3.7)$ & $2(7.7)$ & $1(1.8)$ & $5(11.6)$ & $\mathbf{5 3}$ & $\mathbf{0 . 0 3 4 *}$ \\
\hline Focal edema & $19(4.8)$ & $19(6.5)$ & $1(3.9)$ & $6(10.9)$ & $5(11.6)$ & $\mathbf{5 0}$ & 0.203 \\
\hline AIL & $10(2.5)$ & $8(2.7)$ & $1(3.9)$ & $3(5.5)$ & $5(11.6)$ & $\mathbf{2 7}$ & $\varnothing$ \\
\hline Shift & $9(2.3)$ & $4(1.4)$ & $0(0)$ & $0(0)$ & $0(0)$ & $\mathbf{1 3}$ & 0.514 \\
\hline Hemorrhage & $3(0.8)$ & $3(1)$ & $0(0)$ & $3(5.5)$ & $1(2.3)$ & $\mathbf{1 0}$ & $\varnothing$ \\
\hline Hydrocephalus & $1(0.3)$ & $4(1.4)$ & $2(7.7)$ & $0(0)$ & $2(4.7)$ & $\mathbf{9}$ & $\varnothing$ \\
\hline Diffuse edema & $0(0)$ & $2(0.7)$ & $0(0)$ & $0(0)$ & $0(0)$ & $\mathbf{2}$ & $\varnothing$ \\
\hline Arachnoid cyst & $1(0.3)$ & $0(0)$ & $0(0)$ & $0(0)$ & $1(2.3)$ & $\mathbf{2}$ & $\varnothing$ \\
\hline Toplam (n) & $\mathbf{3 9 6}$ & $\mathbf{2 9 4}$ & $\mathbf{2 6}$ & $\mathbf{5 5}$ & $\mathbf{4 3}$ & $\mathbf{8 1 4}$ & \\
\hline
\end{tabular}

Ø: $p$ value could not measured.

Percentage values are the ratio of the row variable to the total number of patients in the column variable.

CSC: Change in seizure characteristics; FS: First seizure; ND: Neurologic deficit; CT: Computed tomography; HBT: High body temperature; SE: Status epilepticus; CIS: Chronic infarct sequelae; DCA: Diffuse cortical atrophy; AIL: Acute ischemic lesion.

\subsection{Characteristics of subjects}

\subsubsection{Age and sex}

$55.7 \%(n=453)$ of the patients were male. The age distribution of the study group was symmetrical, and the mean age was 50 years $(50.50 \pm 20.26$ years, $95 \%$ Confidence Interval). It was observed that the applicants were between the ages of $18-29(n=161,19.8 \%)$.

\subsubsection{Emergency application}

The patients presented to ED frequently in spring, most frequently in April. It was observed that $50.2 \%(n=409)$ of the patients applied to ED via 112 Emergency Health System, $49.1 \%(n=400)$ were as outpatient, and 5 of them were referred.

\subsubsection{Seizure}

$56.5 \%(n=460)$ of the patients presented with chronic seizure activity and $14 \%$ of these patients had changes in the characteristics of seizures. It was found that $58.4 \%$ (n $=475$ ) of the patients had seizures only once, and $60.6 \%$ $(n=493)$ returned to their previous state of consciousness after the seizure. The frequency of refractory seizures was $9.1 \%(n=74)$, of which $60.8 \%(n=45)$ were chronic seizure activity.

$66.5 \%(n=541)$ of the patients had generalized, $20.1 \%$ $(\mathrm{n}=164)$ had focal and $13 \%(\mathrm{n}=106)$ had unclassified seizures. Generalized tonic-clonic was the most common $(\mathrm{n}=471,57.9 \%)$, and focal aware type seizures were the second most common type $(\mathrm{n}=98,12 \%)$. Three patients were in non-convulsive status epilepticus.

During the seizure period, the patients had an average of
2 seizures $(2.18 \pm 0.82$ seizures, $95 \%$ CI). Only $17.8 \%$ (n $=145$ ) of the patients' duration of seizures was determined, and $56.5 \%(\mathrm{n}=82)$ had a seizure for less than 5 minutes.

\subsubsection{Past medical history}

The three most common diseases in past medical histories were previous cerebrovascular disease (CVD), malignancy, and hypertension. The relationships between past medical history and seizure are shown in Table 1.

\subsubsection{Medications}

It was found that $50.4 \%$ of all patients and $18.4 \%$ of those who applied with chronic seizure activity did not receive anticonvulsant medication. Medication information of eighteen $(2.2 \%)$ patients were not found.

Approximately one-third of the patients $(n=243,29.9 \%)$ were using single, and $17.5 \%(\mathrm{n}=143)$ were using combination medication. Valproic acid was the most commonly used agent in patients receiving either a single drug or combined therapy (34.5\% and $20.8 \%$, respectively). Valproic acid+levatiracetam $(\mathrm{n}=19,2.3 \%)$ was the most commonly used dual drug combination.

\subsubsection{Laboratory workup}

Hyponatremia was detected in $21.1 \%(\mathrm{n}=172)$ of patients. Also, hypernatremia was seen in $2.2 \%(n=18)$ of patients. $53.5 \%$ of the patients with hyponatremia were patients with first seizure activity $(\mathrm{n}=92 / 172)$. The incidence of hyponatremia was $17.4 \%(\mathrm{n}=80 / 460)$ in patients with chronic seizure activity and $26 \%(\mathrm{n}=92 / 354)$ in patients with first seizure. Hyponatremia was found significantly higher in 1 out of 4 patients who presented with first seizure 
activity (Pearson Chi-square $\mathrm{p}=0.003$ ).

It was found that $44.4 \%(n=8)$ of the patients with hypernatremia admitted to ED with first seizure activity. The incidence of hypernatremia was $2.3 \%(n=8 / 354)$ in patients with first seizure and $2.2 \%(n=10 / 460)$ in patients with chronic seizure activity. There was no significant difference between the seizure history and the incidence of hypernatremia. (Pearson Chi-square $p=0.936$ ). It was observed that only five patients had no recorded sodium values.

Hypoglycemia was detected in less than $1 \%$ of the patients $(n=6 ; 0.7 \%)$ and hyperglycemia was detected in $57 \%$ $(\mathrm{n}=464)$. Only 2 of 6 hypoglycemic patients were diabetic.

The incidence of hyperglycemia was $65.6 \%(\mathrm{n}=$ 232/354, Pearson Chi-square $p=0.000)$ in patients presented with first seizure and 50.4\% $(n=232 / 460)$ in patients presented with chronic seizure activity. 17.7\% (n $=41 / 232$ ) of the patients with hyperglycemia who have presented with first seizure activity and $5.6 \%(n=13 / 232)$ of the patients with hyperglycemia who have presented with chronic seizure activity were diabetic.

In our study, it was found that only $27.5 \%$ of the patients using antiepileptic drugs had measurements of drug serum concentrations.

\subsection{Main Results}

\subsubsection{Indications for Non-contrast Computed Brain Tomography Imaging}

In our study, the most common indication for imaging was the change in seizure characteristics ( $n=396 ; 48.6 \%$ ), and the second most common indication was first seizure history $(\mathrm{n}=294 ; 36.1 \%)$. The first seizure was the most common indication for emergency $\mathrm{CT}$ imaging in patients presented with first seizure activity $(294 / 354 ; 83.1 \%)$. In patients with chronic seizure activity (396/460; 86.1\%), change in characteristics of seizure was the most common indication for emergency CT imaging.

It was found that the frequency of CT imaging due to status epilepticus and concomitant high fever was higher in chronic seizure patients. $(26 / 460,5.6 \%$ versus $17 / 354$; $4.8 \%$ ) and $(18 / 460 ; 3.9 \%$ versus $8 / 354 ; 2.3 \%)$, respectively.

On the other hand, the frequency of emergency CT imaging because of neurological deficit was higher in patients presented with first seizure activity $(35 / 354 ; 9.9 \%$ versus 20/460; 4.3\%).

\subsubsection{Relationship between the CT Interpretations and seizure histories}

\section{First Seizure}

$59.3 \%(n=210 / 354)$ of the patients who were admitted to ED for the first seizure activity had abnormal findings in their CT interpretations. More than half of these findings $(57.1 \% ; n=120 / 210)$ were new. The occurance incidence of new abnormal CT findings in patients presenting to ED because of first seizure was $33.9 \%(n=120 / 354)$. (Fig. 1).

The most common abnormal CT findings in patients presenting with the first seizure were diffuse cortical atrophy
( $\mathrm{n}=84 ; 23.7 \%)$ and chronic infarct sequelae $(\mathrm{n}=84$; 23.7\%). Diffuse cortical atrophy and mass findings were significantly higher in patients who underwent brain CT imaging for the first seizure activity (Fisher's Exact Test $\mathrm{p}=0.013$ and Fisher's Exact Test $\mathrm{p}=0.024$, respectively) (Table 2).

\section{Chronic seizure activity}

$46.5 \%(n=214 / 460)$ of the patients who presented with chronic seizure activity to ED had abnormal CT findings. $25.2 \%(n=54 / 214)$ of these patients had new findings. The occurrence incidence of new abnormal CT findings in patients presented with chronic seizure activity to ED was $11.7 \%(n=54 / 460)$ (Fig. 2).

Chronic infarct sequelae $(n=95,20.6 \%)$ was the most common abnormal finding in brain CTs of patients presented with chronic seizure activity. Operation sequelae findings were significantly higher in patients with chronic seizure activity (Fisher's Exact Test $\mathrm{p}=0.010$ ) (Table 2).

The incidence of abnormal CT findings was $52 \%(\mathrm{n}=$ $424 / 814$ ) in the study. Of these abnormal findings, $25.7 \%$ were diffuse (65 in first, 44 in chronic seizure patients), $58.2 \%$ in focal (119 in first, 128 in chronic seizure patients), and $16 \%$ (26 in first and 42 chronic seizure patients) mixed lesions. The most common abnormal CT finding was chronic infarct sequelae $(\mathrm{n}=179,22 \%)$. The second most common was diffuse cortical atrophy $(n=160,19.7 \%)$.

\subsubsection{Relationship between imaging indications and abnormal CT findings}

Mass findings were significantly higher in patients undergoing brain CT imaging for neurological deficit. Similiarly, cortical atrophy findings for the patients undergoing CT imaging because of concomitant high fever, and operation sequelae findings for the patients undergoing CT imaging because of status epilepticus were significantly higher (Table 3 ).

\subsubsection{Patient Outcomes}

$75.3 \%(n=613)$ of the patients were discharged after observation, and $21 \%$ were hospitalized $(n=171 ; 77$ of them were in the ward, and 94 were in the intensive care unit). Twenty-nine patients $(3.5 \%)$ left the ED with treatment refusal. Among the hospitalized patients, intensive care hospitalization rate was $54.9 \%$. In the study group, this was $11.5 \%(n=94 / 814)$. Only one patient $(0.1 \%)$ died in ED (Stage 4 Colon cancer).

It was found that $50.6 \%(\mathrm{n}=87)$ of 172 patients (including exitus) who were hospitalized were presented with a first-time seizure, and $49.4 \%(n=85)$ of them were presented with chronic seizure activity. No significant difference was found between the seizure histories in terms of hospitalization rates.

The mean length of stay in the ED was 537 minutes $(537.7 \pm 352.4$ minutes, $95 \% \mathrm{CI})$. This period was $452 \mathrm{~min}$ utes ( $452 \pm 250.9$ minutes, $95 \% \mathrm{CI}$ ) for patients discharged after observation and 574 minutes $(574 \pm 537.3$ minutes, 


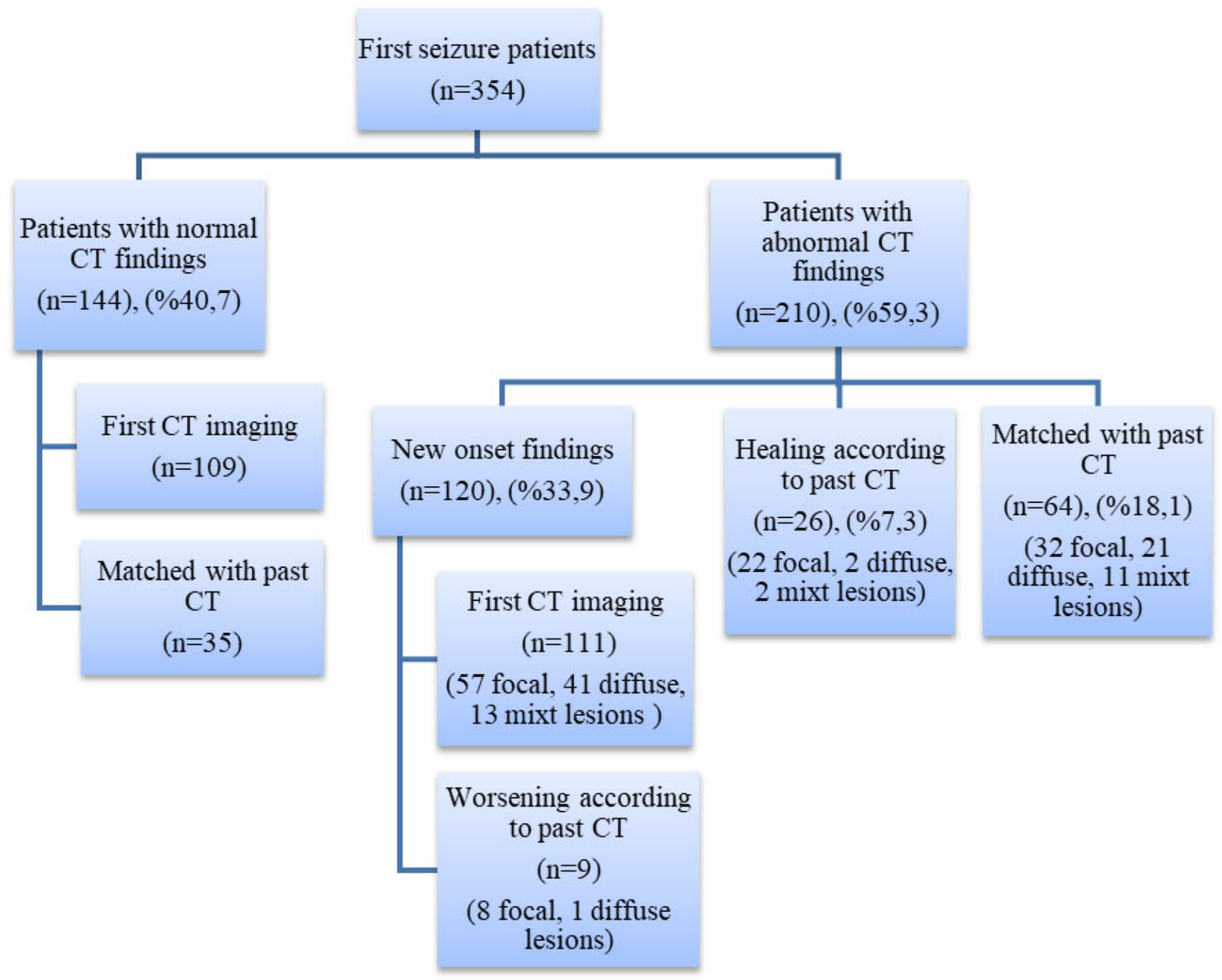

F I G U R E 1. Distribution of computed tomography findings in first seizure patients.

CT: Computed Tomography.

$95 \% \mathrm{CI})$ for hospitalized patients.

There was a significant difference between the two groups in terms of length of stay in the ED (Mann-Whitney $\mathrm{U}$ test $\mathrm{p}=0.000$ ).

Decision of discharge was found to be significantly higher for seizure patients $(\mathrm{p}<0.001 ; \mathrm{n}=172$ vs 642$)$. No relationship was found between the admission and outcome status of the patients.

52 of hospitalized 87 first seizure patients had new abnormal CT findings. We found that medical management of $14.7 \%$ (52/354) of first seizure patients were changed due to new abnormal CT findings. Similarly, in 33 of hospitalized 85 chronic seizure patients had new abnormal CT findings. It was also found that management of $7.2 \%(33 / 460)$ of the chronic seizure activity patients were changed due to new abnormal CT findings.

It was found that the ED management of $10.4 \%$ of all patients $(85 / 814)$ who applied to ED because of seizure was changed due to performing non-contrast $\mathrm{CT}$ imaging in patients.

\section{Discussion}

The seizure is one of the medical conditions that cause at least one ED application until the age of 80 and constitutes approximately $1 \%$ of emergency applications $[2$, $4,5]$. Whether the first episode (unprovoked) due to an acute condition or a chronic recurrent (provoked) history, seizures that cannot be controlled rapidly are considered as a neurological emergency $[4,5,12]$.

Evaluation of important information such as past medical records, medications, concomitant diseases is critical clues in the management and treatment of the patients. Similarly, the duration and progression of seizure are essential in clarifying whether the event is a seizure or a mimicking condition [12]. Witnesses and pre-hospital emergency healthcare professionals are important sources of information. Any history of trauma, the presence of a diagnosis of epilepsy, or the post-seizure period directly affects the diagnosis and treatment [12].

In the literature, various studies have reported that comorbid diseases might cause first seizures or trigger seizures in epilepsy patients $[2,11,12,15,16]$. On the 


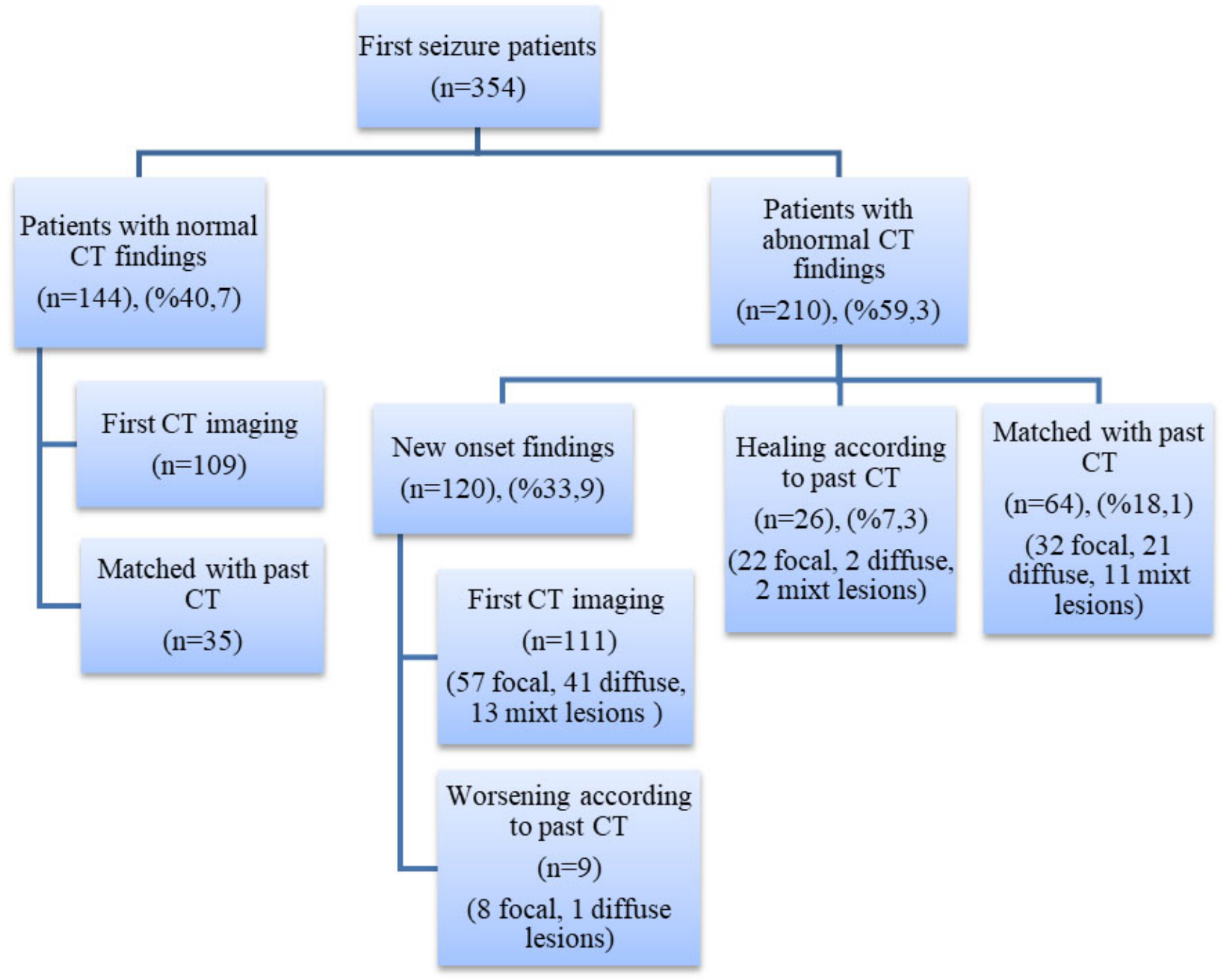

F I G U R E 2. Distribution of computed tomography findings in chronic seizure patients.

CT: Computed Tomography.

other hand, we could not find clear information about which comorbid diseases are more common in first or chronic seizure patients.

In our study, hypertension was the most common disease, but it was found that many comorbid diseases that may cause seizures were also significantly higher in patients who had seizures for the first time. However, in patients with chronic seizure activity, only presence of intracranial operation and mental motor retardation histories were found to be significantly higher as the condition that may cause seizures. (Table 1).

It is reported that the most common cause of ED admissions in chronic seizure patients is incompatible with anticonvulsant medications [3, 12]. Similarly, in our study, it was found that $45.6 \%$ of chronic seizure patients who applied to the ED despite multiple anticonvulsant medication or because of not using their medications.

The American College of Emergency Physicians (ACEP) published its first clinical policy guideline on the management of patients who applied to ED due to seizure in 2004 and did not recommend detailed metabolic tests for patients who returned to their normal neurological status after the first seizure [2]. If the patient presents with seizure for the first time, has no known comorbid disease, and physical examination including neurological examination is normal, the probability of an electrolyte disorder is reported to be extremely low $[2,12]$.

In the same guideline, only serum glucose, sodium levels, and pregnancy test for women in reproductive age are recommended for healthy individuals with normal neurological status presenting with a first seizure. These recommendations were also reported in the guidelines published by the American Neurology Association (ANA) in 2007 on the management of the first seizure in children, and updated ACEP guidelines in 2014 [11, 12, 16].

In our study, hyponatremia and hyperglycemia were found significantly higher in patients who applied to ED for the first seizure. Therefore, we think that hyponatremia and hyperglycemia should be differentiated in the first line as the cause of seizure in the first seizure patients.

Another recommended emergency laboratory work up is to study serum antiepileptic drug level if can be measure, in patients with a history of seizure and present with seizure similar to previous seizures $[10,12]$. 
In our study, it was found that only $27.5 \%$ of patients were evaluated in who required drug serum level measurement. This rate was insufficient to evaluate both drug compliance and drug efficacy. This can be explained by the problems with laboratory resources and the personal medical opinion of the physician.

The number and duration of seizure are important criteria for emergency physicians in the management of seizure patients. Whether it is recurrent or prolonged, it is now known that seizure activity can lead to cognitive impairment. It has also been reported that status epilepticus alone can impair cognitive functions, regardless of the cause that initiated the seizure [12].

In light of current literature, the idea has been adopted that worsening of epilepsy, and cognitive impairment and intellectual disability often develops due to the seizure mechanism.

In our study, the rate of patients whose consciousness did not improve after seizures or between seizures was $9.1 \%$. It was observed that this group of patients frequently had refractory seizures and status epilepticus. We could not come across any clear information about the number or duration of seizures observed during the attack period in the literature. We found that $58.4 \%$ of the patients had seizures only once during the first attack. Also, even if seizure was intervened with antiepileptic $\operatorname{drug}(\mathrm{s})$, the avarage number of seizures was 2 . The mean seizure duration of the study group could not be determined because the seizure durations were not clearly known.

The need and timing of neuroimaging in patients with first seizure is a controversial issue for emergency physicians [13, 17-20]. Although neurologists tend to prefer MRI to CT, no precise published data is showing the superiority of MRI to CT [10]. ANA suggested that computed tomography is the first appropriate imaging modality in patients with first seizure and even in some cases of chronic seizure activity $[6,10,12]$.

In the presence of contraindications for MRI (such as pacemaker, non-compatible aneurysm clips, and orthopedic or cardiac implants, etc.), or in the absence of MRI ability, brain CT was defined as a usefull tool in the ED to clarify hemorrhage (traumatic/non-traumatic), mass lesions and large infarcts and their complications [4, 5, 7, 9].

The criteria that can be used for determining which patients may benefit from emergency CT, among patients who applied to ED for the first seizure, have been defined. These criteria are often described as factors associated with the likelihood of detecting abnormal CT findings [11-13].

These are malignancy [11], history of closed-head injury $[11,12]$, neurocutaneous disorders $[11,12]$, focal onset seizures [11, 12], alcohol abuse and withdrawal [11, 12], history of cysticercosis [12], prolonged unconsciousness [12], seizure activity over age 65 and longer $15 \mathrm{~min}$ [12], persistent fever [13], history of anticoagulant use [13], immune-compromised conditions (e.g., AIDS) [11, 13], and new focal abnormality in neurological examination [11-13].
It has been reported that abnormal $\mathrm{CT}$ findings range between $3-56 \%$ in patients who were evaluated with noncontrast CT because of first time seizure in the ED. [11, 1720]. Similarly, we found an abnormal CT finding in one of two over all patients $(52 \%)$, in our study. The rate of new abnormal findings on emergency CT imaging in first seizure patients was found $33.9 \%$.

In literature, there is no consensus supporting or rejecting the necessity of emergency imaging in patients presenting with chronic seizure activity. Evaluations on this group patients were stated in an evidence-based review that was published in 2007 and that review was based on only 3 class 3 studies. In these studies, it was reported that the rates of abnormal CT findings of patients presenting with first seizure (pediatric and adult) and those presenting with chronic seizure activity were similar, and there was no significant difference between them [11].

In contrast to the literature, in our study, the rate of new abnormal findings on CT imaging of patients who were admitted to the ED due to chronic seizure activity was $11.7 \%$. Also, the rate of new abnormal findings in noncontrast brain CT was up to 3 times higher in patients with first seizure than in patients with chronic seizures $(33.9 \%$ vs. $11.7 \%$ ).

It was reported that two-thirds of the CT findings detected in patients presenting to ED because of first seizure were focal, [11, 17-19] and these were intracranial hemorrhage, brain abscess and tumors [7, 11], with structural malformations and calcified lesions [9, 11], and cortical atrophy and infarcts [20].

Similarly, in our study, $56.7 \%$ of the abnormal CT findings observed in patients with first seizure were also focal lesions. On the other hand, $27.8 \%$ of abnormal CT findings in patients with chronic seizure activity patients were focal lesions.

Diffuse cortical atrophy and mass findings were significantly more common in the first seizure patients, while operation sequelae findings were significantly more common in patients with chronic seizure activity. We think that the most important reason for this result may be that pediatric patients who have different seizure etiology were not evaluated.

It was reported that emergency management of $9-17 \%$ of patients, who admitted to ED because of first seizure, was changed due to pathological findings which detected by brain CT [7, 9, 11, 12, 16, 20]. Similarly, in our study, the rate of change in management of patients who presented because of first seizure was determined as $14.7 \%$ due to pathological findings in brain $\mathrm{CT}$.

Contrary to this, no study demonstrating the possibility of emergency management change due to neuroimaging of the chronic seizure patients who present to ED with seizure activity has been reported [11].

In our study, the rate of chronic seizure patients whose emergency managements were changed due to new abnormal findings on non-contrast CT imaging, that has not been mentioned in the literature, was found to be $7.2 \%$. 
In the literature, clear statements about the conditions under which the first seizure patients can be discharged from ED have been reported [12]. However, there is no clear information about how long these patients should be observed in ED [4, 20]. In this study, it was observed that the mean length of stay in ED patients who had been admitted to the inpatient clinic was 120 minutes longer than that of outpatients and patients who were discharged. This difference was thought to be due to prolonged consultation procedures and transfer time to the inpatient clinic.

\section{Conclusions}

An abnormal finding was detected in one out of two patients who applied to ED for seizure and evaluated with noncontrast CT imaging. Also, abnormal CT findings were observed in one-third of the patients who presented to ED because of first seizure. It was found that these findings changed the management of $14.7 \%$ of the patients with a first seizure.

One out of every ten patients who presented to ED with chronic seizure activity had a new abnormal CT finding. It was also found that these findings changed the management of $7.2 \%$ of the patients with chronic seizure activity.

It was found that the emergency management of all seizure patients who underwent emergency non-contrast brain CT imaging was changed by $10.4 \%$ due to newly developed CT findings.

Non-contrast brain CT imaging is a diagnostic method that can be used in the differential diagnosis of patients presenting with seizures to ED, whether the first or chronic seizures, and it could change the emergency management of the patients.

\section{Limitations}

Inadequacies and lack of information in the medical records of the patients caused limitations in accessing certain data. For example, information such as the number and duration of seizures, whether or not there was a first seizure history, could be evaluated as far as the statements of the witnesses and the observations of the doctors recorded in the medical records.

Even if the brain CT interpretations were performed by radiologists, the fact that they were interpreted in emergency conditions might have affected the accuracy and reliability of the interpretation.

\section{ACKNOWLEDGEMENTS}

We would like to thank Okan Sayan for language editing.

\section{CONFLICT OF INTEREST}

The authors declare that there is no conflict of interest regarding the publication of this article.

\section{AUTHOR CONTRIBUTIONS}

MS and GC conceived the study, designed the trial, and obtained research funding. MS and GC supervised the conduct of the trial and supervised the data collection. YE, NYO, IT and RT managed the patients' data, including quality control. MY provided statistical advice on study design and analyzed the data; MS chaired the data oversight committee. GC and MS were drafted the manuscript, and all authors contributed substantially to its revision. MS takes responsibility for the paper as a whole.

\section{ETHICAL APPROVAL}

Ethical approval was obtained from The Local Institutional Ethical Committee (Approval date and no; 30.06.2015/28).

\section{FUNDING INFORMATION}

This research did not receive any specific grant from any funding agency in the public, commercial, or not-for-profit sectors.

\section{INFORMED CONSENT}

Informed consent was not applicable for this present study because of to be retrospective.

\section{HUMAN RIGHTS}

Our work does not infringe on any rights of others, including privacy rights, and intellectual property rights. There is no human rights violation in our manuscript.

\section{AVAILABILITY OF DATA AND MATERIALS}

The datasets analyzed during the current study are available from corresponding author.

\section{REFERENCES}

[1] Hauser WA, Annegers JF, Rocca WA. Descriptive epidemiology of epilepsy: Contributions of population-based studies from Rochester, Minnesota. Mayo Clin Proc. 1996;71:576-586.

[2] American College of Emergency Physicians Clinical Policies Committee; Clinical Policies Subcommittee on Seizures. Clinical policy: critical issues the evaluation and management of adult patients presenting to the emergency department with seizures. Ann Emerg Med. 2004;43:605-625.

[3] Hayden RS. Seizures. In: Mahadevan SV, Garmel GM, editors. An Introduction to Clinical Emergency Medicine: Guide for Practitioners in the Emergency Department. 1st Ed. New York-USA: Cambridge University Press; 2005.p.473-484.

[4] Lung DD, Catlett CL, Tintinalli JE. Seizures and Status Epilepticus in Adults. In: Tintinalli JE, Kelen GD, Stapczynski JS, Eds. A Comprehensive Study Guide: Textbook of emergency medicine. 7th Ed. North Carolina-USA: Mc Graw-Hill; 2010.p.341-352.

[5] Hess CP, Barkovich AJ. Seizures: emergency neuroimaging. Neuroimaging Clin N Am. 2010;20:619-37. 
[6] Krumholz A, Wiebe S, Gonseth G, Shinnar S, Levisohn P, Ting T, et al. Quality Standards Subcommittee of the American Academy of Neurology; American Epilepsy Society. Practice Parameter: evaluating an apparent unprovoked first seizure in adults (an evidencebased review): report of the Quality Standards Subcommittee of the American Academy of Neurology and the American Epilepsy Society. Neurology. 2007;69:1996-2007.

[7] Schachter SC. Evaluation of the first seizure in adults. 2016. accessable on: http://www . uptodate.com/contents/evaluationof-the-first-seizure-in-adults? source=see_link\#H25.

${ }^{[8]}$ Fountain NB, Van Ness PC, Swain-Eng R, Tonn S, Bever CT Jr. Quality improvement in neurology: AAN epilepsy quality measures: Report of the Quality Measurement and Reporting Subcommittee of the American Academy of Neurology. Neurology. 2011;76:94-9.

[9] Haider HA, Hirsch LJ. Neuroimaging in the evaluation of seizures and epilepsy. 2016. accessable on: http: //www . uptodate. com/contents/neuroimaging-in-theevaluation-of-seizures-and-epilepsy .

[10] Hernandez R, Silverberg M. Treatment of seizures in the emergency department: pearls and pitfalls. 2015. accessable on: http://www. emdocs.net/treatment-of-seizures-inthe-emergency-department-pearls-and-pitfalls/.

[11] Harden CL, Huff JS, Schwartz TH, Dubinsky RM, Zimmerman RD, Weinstein S, et al. Reassessment: neuroimaging in the emergency patient presenting with seizure (an evidence-based review): report of the Therapeutics and Technology Assessment Subcommittee of the American Academy of Neurology. Neurology. 2007;69:1772-1780.

[12] Teran F, Harper-Kirskey K, Jaqoda A. Clinical Decision Making In Seizures and Status Epilepticus. Emerg Med Pract. 2015;17;1-24.

[13] ACEP NOW. The Offical Voice of Emergency Medicine. Best Practices for Seizure Management in the Emergency Department. 2011. accessable on: http://www.acepnow.com/article/ best-practices-seizure-management-emergencydepartment/?singlepage $=1$ \& theme=print - friendly.

[14] Glauser T, Shinnar S, Gloss D, Alldredge B, Arya R, Bainbridge J, et al. Evidence-Based Guideline: Treatment of Convulsive Status Epilepticus in Children and Adults: Report of the Guideline Committee of the American Epilepsy Society. Epilepsy Curr. 2016;16:48-61.
[15] Pallin DJ, Goldstein JN, Moussally JS, Pelletier AJ, Green AR, Camargo CA Jr. Seizure visits in US emergency departments: epidemiology and potential disparities in care. Int $\mathbf{J}$ Emerg Med. 2008;1:97-105.

[16] Huff JS, Melnick ER, Tomaszewski CA, Thiessen MEW, Jagoda AS, Fesmire FM. Clinical policy: Critical issues in the evaluation and management of adult patients presenting to the emergency department with seizures. Ann Emerg Med. 2014;63:437-447.

[17] Steering Committee on Quality Improvement and Management, Subcommittee on Febrile Seizures American Academy of Pediatrics. Febrile seizures: clinical practice guideline for the long-term management of the child with simple febrile seizures. Pediatrics. 2008;121:1281-6.

[18] Henneman PL, Deroos F, Lewis RJ. Determining the need for admission in patients with new-onset seizures. Ann Emerg Med. 1994;24:1108-14.

[19] Tardy B, Lafond P, Convers P, Page Y, Zeni F, Viallon A, et al. Adult first generalized seizure: etiology, biological tests, EEG, CT scan, in an ED. Am J Emerg Med. 1995;13:1-5.

[20] Dunn MJ, Breen DP, Davenport RJ, Gray AJ. Early management of adults with an uncomplicated first generalized seizure. Emerg Med J. 2005;22:237-242.

[21] Reinus WR, Wippold FJ 2nd, Erickson KK. Seizure Patient Selection for Emergency Computed-Tomography. Ann Emerg Med. 1993;22:1298-1303.

[22] Dhuna A, Pascual-Leone A, Langendorf F, Anderson DC. Epileptogenic Properties of Cocaine in Humans. Neurotoxicology. 1991;12:621-626.

How to cite this article: Mustafa Sever, Gulsenem Cicek, Necmiye Yalcin Ocak, Yesim Eyler, Rana Toktas, Ibrahim Toker, Murat Yesilaras. Non-contrast Cranial Computed Tomography can Change the Emergency Management of Nontraumatic Seizure Patients. Signa Vitae. 2020;16(1):105-114. doi:10.22514/sv.2020.16.0014. 